\title{
FTY720 for cancer therapy (Review)
}

\author{
LI ZHANG, HAN-DONG WANG, XIANG-JUN JI, ZI-XIANG CONG, JIAN-HONG ZHU and YUAN ZHOU
}

Department of Neurosurgery, Jinling Hospital, School of Medicine, Nanjing University, Nanjing, Jiangsu 210002, P.R. China

Received July 29, 2013; Accepted September 16, 2013

DOI: $10.3892 /$ or.2013.2765

\begin{abstract}
Amino-2-[2-(4-octylphenyl)]-1,3-propanediol hydrochloride (FTY720) is a potent immunosuppressant which has been approved by the Food and Drug Administration (FDA) as a new treatment for multiple sclerosis. As an immunosuppressant, it displays its anti-multiple sclerosis, immunosuppressive effects by activating sphingosine-1-phosphate receptors (S1PRs). In addition to the immunosuppressive effects, FTY720 also shows preclinical antitumor efficacy in several cancer models. In most cases, phosphorylation of FTY720 is not required for its cytotoxic effect, indicating the involvement of S1PR-independent mechanisms which are starkly different from the immunosuppressive property of FTY720. In the present study, we reviewed the rapidly advancing field of FTY720 in cancer therapy as well as some molecular targets of the unphosphorylated form of FTY720.
\end{abstract}

\section{Contents}

1. Introduction

2. Anticancer effects of FTY 720

3. Side-effects

4. Molecular targets of unphosphorylated form of FTY720

5. Conclusion

\section{Introduction}

2-Amino-2-[2-(4-octylphenyl)]-1,3-propanediol hydrochloride (FTY720), also known as fingolimod, is a synthetic compound produced by modification of a metabolite from Isaria sinclairii $(1,2)$. FTY720 is a newly developed immunosuppressant. This drug selectively reduces the number of lymphocytes in the peripheral circulation, significantly prolongs the survival of experimental animals and transplanted organs

Correspondence to: Professor Han-Dong Wang, Department of Neurosurgery, Jinling Hospital, School of Medicine, Nanjing University, 305 East Zhongshan Road, Nanjing, Jiangsu 210002, P.R. China

E-mail:njhdwang@hotmail.com

Key words: FTY720, cancer, molecular targets without prejudice to the immune response to the virus and immune memory function (3-7). In addition, on September 22, 2010, FTY720 became the first oral disease-modifying drug approved by the Food and Drug Administration (FDA) to reduce relapses and delay disability progression in patients with relapsing forms of multiple sclerosis. The immunosuppressive activity of FTY720 has been suggested to be related to its phosphorylation by sphingosine kinase 2 (SphK2) and subsequent modulation of $\mathrm{G}$ protein-coupled sphingosine1-phosphate receptors (S1PRs) (S1PR1, S1PR3, S1PR4, S1PR5) that induce lymphopenia by altering lymphocyte trafficking (8). In addition to the potent immunosuppressive effects, evidence suggests that FTY720 has antitumor efficacy in multiple types of cancer, including breast (9), glioblastoma (10), prostate (11), lung (12), ovarian (13) and hematopoietic malignancies (14). This antitumor activity of FTY720 is reportedly independent of the phosphorylation of FTY720, which is different from the immunosuppressive effects of FTY720 (15). In the present study, we described the latest progress of FTY720 in cancer therapy (Table I).

\section{Anticancer effects of FTY720}

In vitro therapeutic activity of FTY720

Caspase-dependent apoptotic pathway. During studies assessing the lymphopenic action of FTY720, it was revealed that FTY720 also induced apoptosis in peripheral lymphocytes. This observation led to investigations into the potential of FTY720 to act as an apoptosis-inducing anticancer agent (8). FTY720 was first reported to induce early apoptosis in an androgen-independent prostate cancer cell line dependent on caspase-3 activation (16). Subsequently, it demonstrated preclinical antitumor efficacy in various cancer models. The antitumor activity of FTY720 was reportedly attributed to different apoptotic pathways. Generally, there are two distinct pathways for inducing apoptosis, including the mitochondrial death pathway (intrinsic pathway of apoptosis) and the death receptor pathway (extrinsic pathway of apoptosis). The mitochondrial death pathway is controlled by members of the Bcl-2 family, including Bcl-2, Bad, Bax, Bid and Btf proteins on the mitochondrial membrane. Death stimuli increase mitochondrial permeability and release cytochrome $c$ and other factors from the mitochondria, resulting in caspase- 3 activation and then apoptosis. By contrast, the death receptor pathway is mediated by Fas (CD95) and Fas-ligand. Binding of Fas and Fas-ligand induces activation of the caspase cascade via activation of caspase- 8 and results in apoptosis through activation 
Table I. Effects of FTY720 on cancer.

\begin{tabular}{|c|c|c|c|c|}
\hline Cancer & In vitro/in vivo & Molecular targets & Functions & (Ref) \\
\hline Multiple myeloma & In vitro & ROS & $\begin{array}{l}\text { Autophagic cell death and } \\
\text { caspase-dependent apoptosis }\end{array}$ & $(21,26)$ \\
\hline Mantle cell lymphoma & Both & $\begin{array}{l}\text { CD74, Cyclin D1, } \\
\text { PKB }\end{array}$ & $\begin{array}{l}\text { Lysosomal membrane permeabilization } \\
\text { related cell death and prolonged } \\
\text { survival in mouse }\end{array}$ & $(23,28)$ \\
\hline Acute lymphoblastic leukemia & In vitro & ROS & Caspase, PP2A-independent cell death & (25) \\
\hline $\begin{array}{l}\text { Chronic lymphocytic leukemia } \\
\text { and lymphoblastic leukemia/ } \\
\text { lymphoma }\end{array}$ & Both & PP2A, ERK1/2 & $\begin{array}{l}\text { Induce caspase-independent cell death and } \\
\text { prolong survival in mouse models }\end{array}$ & (24) \\
\hline Glioma & Both & FAK, ERK $1 / 2$ & $\begin{array}{l}\text { Induce caspase-dependent apoptosis, } \\
\text { decrease invasion and inhibit tumor } \\
\text { growth, increase survival time in } \\
\text { mouse models }\end{array}$ & $(35,36)$ \\
\hline Lung carcinoma & Both & S1P, CXCR4 & Antiangiogenic and reduce tumor size & $(12,37,38)$ \\
\hline Neuroblastoma & Both & SphK2, AKT & $\begin{array}{l}\text { Induce cell death and inhibited } \\
\text { the growth of NB xenografts }\end{array}$ & (39) \\
\hline Prostate carcinoma & In vitro & $\begin{array}{l}\text { p38MAPK, ERK1/2, } \\
\text { FAK, SphK } 1\end{array}$ & $\begin{array}{l}\text { Induce caspase-dependent apoptosis } \\
\text { and sensitize cells to radiotherapy }\end{array}$ & $(40-42)$ \\
\hline Hepatocellular carcinoma & Both & $\begin{array}{l}\text { Rac, EPCs, CXCL10, } \\
\text { VEGF }\end{array}$ & Suppress metastasis & $(43,44)$ \\
\hline Breast cancer & Both & SphK1, JNK & $\begin{array}{l}\text { Induce apoptotic cell death, prevent } \\
\text { tumor growth, metastasis and } \\
\text { prolong animal survival }\end{array}$ & $(9,42,45,46)$ \\
\hline Gastric cancer & Both & PTEN, p53, AKT & $\begin{array}{l}\text { Induce caspase-dependent apoptosis } \\
\text { and inhibit tumor growth }\end{array}$ & (19) \\
\hline Pancreatic cancer & In vitro & AKT & $\begin{array}{l}\text { Induce caspase-dependent apoptosis, } \\
\text { suppress invasion and migration }\end{array}$ & $(47,48)$ \\
\hline Colorectal cancer & In vitro & JNK & Antiproliferative & (46) \\
\hline
\end{tabular}

ROS, reactive oxygen species; PP2A, protein phosphatase 2A; CD74, cluster of differentiation 74; PKB, protein kinase B; ERK1/2, extracellular signal-regulated kinase; FAK, focal adhesion kinase; S1P, sphingosine 1-phosphate; CXCR4, C-X-C chemokine receptor type 4; SphK2, sphingosine kinase 2; p38MAPK, p38 mitogen-activated protein kinase; JNK, c-Jun N-terminal kinase; SphK1, sphingosine kinase 1; EPCs, endothelial progenitor cells; CXCL10, C-X-C motif chemokine ligand 10; VEGF, vascular endothelial growth factor; PTEN, phosphatase and tensin homolog; p53, tumor protein 53.

of caspase-3 $(17,18)$. It has been demonstrated that FTY720 could activate caspase-3, caspase-9 and poly (ADP-ribose) polymerase (PARP) in gastric cancer cells, however there was no obvious change in procaspases- 8 and -10 , and the cleaved products were not detected (19). In addition, FTY720 was shown to increase the levels of caspase-2 and -3 and apoptosis-promoting Bcl-associated proteins, Bad, Bax, Bid and Btf, and decrease the protein level of Bcl-2 in human renal cancer cells. However, there was no difference in the protein levels of caspase-8, Fas, FADD and TRADD, between cells treated with FTY720 and controls (20). These data indicate that FTY720 induces apoptosis through the intrinsic apoptotic pathways, instead of the extrinsic pathway of apoptosis. By contrast, in multiple myeloma cells, FTY720 triggered activation of caspase-8, $-9,-3$, PARP cleavage, induced alterations in mitochondrial membrane potential and Bax cleavage, and anti-Fas antibodies augmented anti-multiple myeloma activity induced by FTY720 (21), demonstrating that FTY720 enhances death signaling via both the extrinsic and intrinsic pathways. The difference may be due to the intricate connections between FTY720 and cell apoptosis, depending on cell types and stimulation manners. Furthermore, there exists a caspase-independent apoptotic pathway that is mediated by apoptosis-inducing factor (AIF) (22). However, no reports as yet have demonstrated that FTY720 could induce cell apoptosis through this pathway.

Caspase-independent cell death. Due to the differences among cell types, there are also reports showing that FTY720 induces cell death independent of apoptosis. For example, in mantle cell lymphoma, FTY720 mediated time- and 
dose-dependent cytotoxicity in primary MCL tumor cells and MCL cell lines; however, FTY720 failed to mediate caspase-3 activation and the expression of Bcl-2 and Mcl-1, two critical anti-apoptotic proteins in MCL, were not altered in response to FTY720 (23). In another case, FTY720 induced toxicity in the Raji cell line and primary CLL B cells, which was independent of activation of caspases or PARP processing (24). The mechanisms underlying the caspase-independent cell death are extremely complex, and include the downmodulation of cyclin D1 and phospho-Akt (23), activation of protein phosphatase 2A (PP2A), dephosphorylation of extracellular signal regulated kinase (ERK) $1 / 2(24)$, the generation of reactive oxygen species (ROS) $(23,25)$, and the induction of autophagy $(13,25-28)$. However, the precise mechanisms remain unclear and are discussed later.

Autophagy. Numerous studies have shown that FTY720 was able to induce autophagy in cancer cells. Autophagy is a process by which cells conserve and recycle their organelles when in a nutrient-deprived or stressed state (29). One of the most important functions of autophagy is to maintain cellular energy subjected to nutrient deprivation and potentially other forms of stress (30). However, extensive or inappropriate activation of autophagy can lead to cell death (type II programmed cell death). Autophagy begins with the formation of an autophagosome, which travels through the cytoplasm of the cell to a lysosome, and the two organelles fuse, within the lysosome, the contents of the autophagosome are degraded via acidic lysosomal hydrolases (31). It has been demonstrated that FTY720 induced autophagy in ovarian cancer cells $(8,13)$, multiple myeloma cells $(26)$, acute lymphoblastic leukemia cells (25). However, the relationship between FTY720-induced autophagy and apoptosis or cell death is controversial. FTY720 could induce both apoptosis and autophagy in multiple myeloma cells, autophagy induced by FTY720 in multiple myeloma cells promoted apoptosis, as evidenced by bafilomycin A1, an autophagy inhibitor, rescued cell death caused by FTY720 (26). Conversely, in ovarian cancer and acute lymphoblastic leukemia cells, FTY720 induced autophagy and caspase-independent cell death. Blocking autophagy by $3 \mathrm{MA}$, a known inhibitor that blocks the autophagy pathway at early stages, or specific siRNA which knocked down the expression of LC3 and Beclin-1 could further enhance FTY720-induced cytotoxicity, suggesting a protective role of autophagy $(13,25)$. The discrepancies may be due to the complex and diverse interplays between autophagy and cell death. Depending on cell types, environment and stimulus, autophagy and cell death may have inhibitory, additive or even synergistic effects (32). Therefore, we consider the autophagy induced by FTY720 in different cell types may lead to either cell survival or cell death. It should be emphasized that FTY720-induced autophagy is also cell-specific. Although FTY720 induced caspase-dependent apoptosis in both MCF7 breast cancer cells and Huh7 hepatoma cells, no evident induction of autophagy was observed in these cells following FTY720 treatment (13). In addition to the induction of autophagy, FTY720 also induced blockage of autophagy. In mantle cell lymphoma cells, FTY720-treated cells showed characteristics of autophagy blockage, including accumulation of autolysosomes and increased LC3-II and p62 levels, which enhanced anticancer efficacy of milatuzumab $(27,28)$. Thus, the effects of FTY720 on autophagy are complex and further studies are required.

In vivo therapeutic activity of FTY720. In addition to the therapeutic activity in vitro, FTY720 also shows significant treatment effects in animal models. For example, in nude mice with human gastric cancer cell xenografts, the tumor growth and cell proliferation in FTY720-treated mice were significantly suppressed; the apoptotic index was markedly increased in that of FTY720 groups (19). In addition, in mouse breast cancer models, tumor growth and metastasis, which were markedly evident in control mice, were significantly prevented in the FTY720-treated groups, resulting in a significant prolongation of animal survival (9). Moreover, FTY720 showed in vivo anticancer effects in glioblastoma (33), hepatic carcinoma (34), lung cancer (12), renal cancer (20), mantle cell lymphoma (23) and prostate cancer (35). Consequently, FTY720 has promising effects in cancer therapy both in vitro and in vivo.

\section{Side-effects}

In multiple sclerosis models, the used doses of FTY720 are $<0.5 \mathrm{mg} /$ day. However, the doses required for anticancer effects of FTY720 in animal models (5 or $10 \mathrm{mg}$ /day) are higher than those used in multiple sclerosis models. Serious adverse events of FTY720 for multiple sclerosis include bradycardia, relapse, basal-cell carcinoma. Other adverse events include macular edema, cancer and laboratory abnormalities (36). In vivo studies of the effects of FTY720 on cancer are conducted only on mice. In animal cancer models, FTY720 treatment did not cause any severe side-effects at a dosage of $\leq 10 \mathrm{mg} / \mathrm{kg} /$ day in mouse renal cancer models. In this case, all mice survived with a healthy appearance and no loss of body weight during the follow up period when treatment was performed at a dosage of $\leq 10 \mathrm{mg} / \mathrm{kg}$ /day (20). Furthermore, FTY720 prevented tumor growth and metastasis of mouse breast (9) and bladder (37) xenografts in nude mice without causing detectable toxicity in vital organs. However, whether these adverse effects presented in multiple sclerosis would occur during human cancer treatment, or could be tolerated if the therapy is efficacious, remains to be determined.

\section{Molecular targets of unphosphorylated form of FTY720}

The ability of FTY720 to induce cell death in cancer cells appears largely independent of the phosphorylation of the drug. FTY720 was able to induce cell death in ovarian cancer (13) and acute lymphoblastic leukemia cells (25); however, the phosphorylated form of FTY720 (FTY720-P) failed to induce cell death, demonstrating that activation of S1PRS is not sufficient for cell death. While the mechanisms mediating the cell death action of FTY720 have yet to be fully clarified, a number of molecular targets of unphosphorylated form of FTY720 have been proposed which may explain the biological effects of this drug (Fig. 1).

ROS. ROS are chemically reactive molecules containing oxygen. Examples include oxygen ions and peroxides. ROS form as a natural byproduct of the normal metabolism of oxygen 


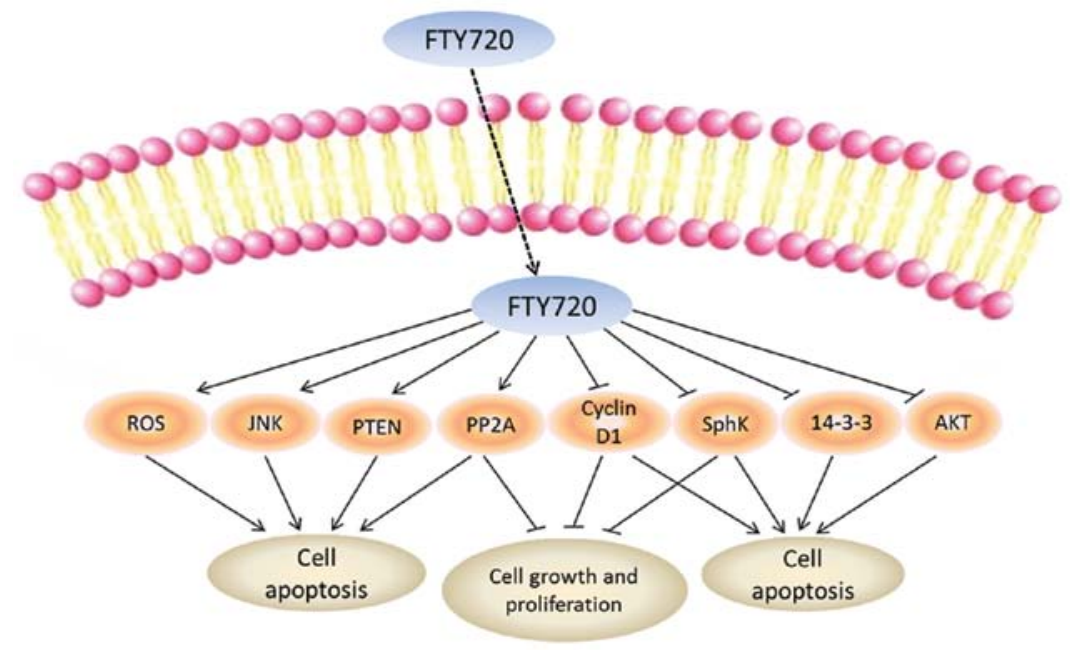

Figure 1. Molecular targets of unphosphorylated form of FTY720. Uptake of FTY720 into the cell leads to its direct activation of reactive oxygen species (ROS), protein phosphatase 2A (PP2A), c-Jun N-terminal kinase (JNK), phosphatase and tensin homolog (PTEN), while inhibiting cyclin D1, sphingosine kinase (SphK), protein 14-3-3, protein kinase B (PKB or AKT). Targeting these molecular targets may result in apoptosis of cancer cells. Moreover, activation of PP2A and inhibition of cyclin D1, SphK can suppress cell growth and proliferation.

and have important roles in cell signaling and homeostasis (38). However, under oxidative stress conditions, excessive ROS can damage cellular proteins, lipids and DNA, leading to fatal lesions in cells that contribute to carcinogenesis.

In acute lymphoblastic leukemia cells, FTY720 increased ROS generation in a time-dependent manner, which then resulted in a caspase-independent cell death, and the antioxidant NAC could block the cell death induced by FTY720 (25). The same occurred in mantle cell lymphoma (23), indicating that ROS is an upstream of cell death induced by FTY720. However, in multiple myeloma cells, FTY720-induced ROS activity promoted autophagy, reduced the expression of Mcl-1, survivin, bcl-2 and increased cleavage of Bid, ultimately leading to apoptosis of multiple myeloma cells (26). Furthermore, in hepatic carcinoma cells, ROS generation induced by FTY720 culminated in protein kinase $\mathrm{C}$ (PKC) $\delta$ activation and subsequent caspase-3-dependent apoptosis (39). In these two cases, ROS generated by FTY720 promoted cell apoptosis rather than death. Thus, the difference demonstrates that ROS may be just a mediate between FTY720 and apoptosis or cell death. However, whether FTY720 induces apoptosis or cell death depends on cell types. Of note, OSU-2S, a non-immunosuppressive analogue of FTY720, increased ROS generation, suppressed cell growth in the same way as FTY720 in hepatocellular carcinoma (40), suggesting a direct effect on ROS of unphosphorylated form of FTY720.

Protein phosphatase 2A (PP2A). PP2A is a ubiquitous and conserved serine/threonine phosphatase with broad substrate specificity and diverse cellular functions. PP2A has been shown to be genetically altered or functionally inactivated in several types of solid cancer and leukemias, and is therefore a tumor suppressor. Changes in PP2A subunits or loss of phosphatase activity have been linked to cancer development and to non-neoplastic diseases (41).

Currently, several studies have demonstrated that the anticancer activity of FTY720 may depend on its ability to act as a potentPP2A activator.Inovariancancercells,FTY720activated
PP2A, decreased autophagy and increased cell death (42). In this case, autophagy acted in a protective role. In acute myeloid leukemia (AML), FTY720-induced PP2A activity resulted in a dose-dependent growth inhibition and a significant decrease in viable cells. The specific PP2A inhibitor OA partially rescued the cells from FTY720-induced apoptosis (43), indicating that PP2A activation is required for FTY720-induced apoptosis in AML cells. FTY720 also induced cell apoptosis in lung tumour (44), CML and Ph-positive B-ALL progenitors $(45,46)$ potentially by disrupting SET-PP2A interaction, allowing PP2A activation. Furthermore, animal studies demonstrated that FTY720 has anticancer effects in vivo by activating PP2A. Reports highlighted the efficacy of FTY720 in animal models of AML, reporting restored PP2A activity, decreased clonogenicity, and suppression of disease $(47,48)$. However, it is unclear whether the anticancer effects of FTY720 in some malignancies should be attributed to activation of PP2A or to other mechanisms (such as autophagy) (41). For example, although FTY720 induced PP2A activation and cell death in acute lymphoblastic leukemia cells, activation of PP2A is not required for FTY720-induced cell death (25).

Mitogen-activated protein kinase (MAPK) signaling pathway. MAPKs are serine/threonine-specific protein kinases belonging to the CMGC (CDK/MAPK/GSK3/CLK) kinase group (49). They regulate proliferation, gene expression, differentiation, mitosis, cell survival, and apoptosis in cancer cells. There are several members in the MAPKs family, among which the ERKs and c-Jun N-terminal kinases (JNKs) are the most important in regulating cell death and survival.

FTY720 has recently been shown to induce the activation of ERK1/ERK2 and JNK1/JNK2, but not p38 in breast and colon cancer cells, which may result in the anti-proliferative activity of FTY720 (50). Furthermore, FTY720 increased phosphorylation of ERK in mantle cell lymphoma (23), but decreased phosphorylation of ERK in glioblastoma (33) and renal cancer cells (20); notably, they all finally contributed to apoptosis of cancer cells. The mediators between FTY720 and 
the MAPK signaling pathway have not been fully clarified, but recent literature indicated that ROS may be involved in the activation of the MAPK pathway. For example, curcumin induced phosphorylation of ERK1/2 and p38 MAPK, yet the curcumin-induced phosphorylation of ERK1/2 and p38 MAPK was attenuated in the presence of NAC, a scavenger of ROS (51). Furthermore, piperlongumine selectively killed glioblastoma cells via JNK and p38 activation, and these activations were blocked by NAC pre-treatment (52). Thus, FTY720 may also activate MAPK signaling pathway through ROS generation, but, to date, how FTY720 mediates MAPK has not been studied. Although the relationship between FTY720 and MAPK signaling pathway remains unclear, the MAPK signaling pathway may be a pivotal target of FTY720 in cancer therapy.

PI3K/AKT/mTOR signaling pathway. PI3K/Akt/mTOR is another important intracellular signaling pathway in regulating cell survival and death. The mammalian target of rapamycin (mTOR) is a serine/threonine protein kinase which works downstream of protein kinase B (Akt). Once activated, mTOR triggers the phosphorylation of the downstream target p70S6K1, enhances the transcription of certain mRNAs, and increases the expression of proteins associated with proliferation (53-55). It has been reported that the PI3K/Akt/mTOR signaling pathway is overactivated in several types of cancer, such as non-small cell lung cancer $(56,57)$, breast cancer $(58)$, colorectal cancer (59) and cholangiocarcinoma (60). Therefore, some experimental cancer drugs aim to inhibit the signaling sequence at some point (61-63).

FTY720 could also facilitate PI3K/AKT signaling pathway to induce cell death. In gastric cancer cells, FTY720 induced apparent decrease of phosphorylation of AKT (473) level, followed by a concentration-dependent reduction of Bcl-2, a concomitant increase of Bax, cleavage caspase-3, -9, PARP, resulting in significant apoptosis in cancer cells (19). Moreover, FTY720 induced p-AKT downmodulation in mantle cell lymphoma, which then caused cell death $(23,28)$. In addition, in prostate cancer (64), neuroblastoma (65) and glioma $(10,66)$ cells, FTY720 also showed anticancer effects by downregulating the phosphorylation of AKT.

Cyclin D1. Cyclin D1 belongs to the highly conserved cyclin family. It functions as a regulatory subunit of CDK4 or CDK6, whose activity is required for cell cycle G1/S transition. This protein has also been shown to interact with tumor suppressor protein $\mathrm{Rb}$ and the expression of cyclin $\mathrm{D} 1$ is regulated positively by $\mathrm{Rb}$. Mutations, amplification and overexpression of this protein, which alters cell cycle progression, are frequently observed in a variety of tumors and may contribute to tumorigenesis. Cyclin D1 has been reported to be involved in tumorigenesis in breast cancer (67), prostate cancer (68), colorectal cancer $(59,69)$, ovarian carcinoma $(70)$, non-small cell lung cancer (71) and gastric carcinoma (72) cells.

Recently, studies showed that in mantle cell lymphoma $(23,28)$, human hepatoma cells (14), rat glomerular mesangial cells (73) and mouse skin transplantation models (74), FTY720 reduced the protein level of cyclin D1, induced G1 phase cell cycle arrest, eventually inhibited cell proliferation and induced cell death. Meanwhile, recent data have also indicated a role for cyclin D2 and cyclin D3 in the pathogenesis of cancer (75-81); however, no reports have studied the effects of FTY720 on cyclin D2 or cyclin D3 and, thus, remains to be explored.

Phosphatase and tensin homolog (PTEN). PTEN is a protein that, in humans, is encoded by the PTEN gene (82). It is one of the most commonly lost tumor suppressors in human cancer. During tumor development, mutations and deletions of PTEN occur that inactivate its enzymatic activity leading to increased cell proliferation and reduced cell death. Frequent genetic inactivation of PTEN occurs in glioblastoma $(83,84)$, endometrial cancer (85), and prostate cancer (86), and reduced expression is found in numerous other tumor types such as lung (87) and breast cancer (88).

However, studies on the effects of FTY720 on PTEN are few and only one reported that FTY720 induced obvious PTEN expression, consistent with a substantial decrease in p-Akt and MDM2, finally inhibiting gastric cancer cell proliferation, inducing G1 phase cell cycle arrest and apoptosis. Suppression of PTEN expression with siRNA significantly activated Akt, resulting in decreased apoptosis and increased cell survival (19). Thus, whether the anticancer activity of FTY720 in some malignancies is related to downmodulation of PTEN remains to be clarified.

Sphingosine kinase (SphK). SphK is a conserved lipid kinase that catalyzes formation sphingosine-1-phoshate (S1P) from the precursor sphingolipid sphingosine. There are two forms of SphK, SphK1 and SphK2.

SphK1 is a lipid enzyme with oncogenic properties that converts the proapoptotic lipids ceramide and sphingosine into the antiapoptotic lipid S1P and activates the signal transduction pathways that lead to cell proliferation, migration, the activation of the inflammatory response, and the impairment of apoptosis (89). There is compelling evidence that SphK1 activation contributes to tumorigenesis in prostate cancer (90), colorectal cancer (91) and breast cancer (92) cells. However, until recently, the absence of clinically applicable SphK1 inhibitors limited the translation of these findings into patients (89). Recent studies shed further insight into the mode of action of FTY720 by demonstrating that it directly inhibits SphK1 activity both in vitro and in vivo. For instance, FTY720 could resensitize human colorectal cancer to cetuximab by inhibiting SphK1 both in vitro and in vivo, with inhibition of tumor growth, interference with signal transduction, induction of cancer cell apoptosis, and prolongation of mouse survival (91). In addition to directly inhibiting SphK1 activity, FTY720 has been demonstrated to induce SphK1 degradation via ubiquitination and subsequent proteasomal processing (93); however, the precise mechanism has not been defined.

There exist two mammalian isoforms of SphK, SphK1 and SphK2, which share a considerable degree of sequence similarity, but differ in their developmental expression, subcellular localization and ability to phosphorylate artificial substrates (94). Moreover, FTY720 becomes phosphorylated mostly by SphK2. In neuroblastoma, FTY720, but not P-FTY720, induced neuroblastoma cell death, inhibited the growth of neuroblastoma xenografts and enhanced the tumor-suppressive effect of topotecan both in vitro and in vivo. Notably, FTY720 significantly decreased SphK2 mRNA and 
protein expression instead of SphK1, disrupted the ceramidesphingosine-S1P balance (65). This may be due to the fact that neuroblastoma predominantly expresses SphK2 (95) and overexpression of SphK2 in neuroblastoma cells promoted tumor growth and cell proliferation.

Thus, FTY720 appears to target SphK via multiple mechanisms. With the well described role of SphK in oncogenesis, such effects of FTY720 on this enzyme provide a mechanistic explanation for its anticancer properties.

14-3-3. 14-3-3 proteins are a family of conserved regulatory molecules expressed in all eukaryotic cells. 14-3-3 proteins have the ability to bind a multitude of functionally diverse signaling proteins, including kinases, phosphatases and transmembrane receptors. For example, 14-3-3 interacted with both the dynein intermediate chain (DIC) and an Hsp70 co-chaperone Bcl-2-associated athanogene 3 (BAG3), thereby recruiting chaperone-associated protein cargos to dynein motors for their transport to aggresomes (96). Through binding to apoptosis signal-regulating kinase-1 (ASK-1), 14-3-3 negatively regulated the kinase activity of ASK-1, thereby blocking activation of stress-activated protein kinases (SAPK) such as p38 and JNK (97).

It was recently shown that FTY720 straightly bound to 14-3-3 proteins to render them phosphorylatable on Ser58 (in $14-3-3 \xi$ ) at the dimer interface. Phosphorylation of 14-3-3 induced by FTY720 could facilitate, at least in part, the apoptotic activity of this drug, as cells expressing nonphosphorylatable 14-3-3 exhibited attenuated apoptosis in response to FTY720 (98). Therefore, 14-3-3 proteins are key intermediates linking FTY720 and mitochondria, apoptotic commitment.

\section{Conclusion}

FTY720 was originally developed as an immunosuppressive agent and is currently undergoing multiple clinical trials, including prevention of kidney graft rejection and treatment of relapsing multiple sclerosis. However, over the past ten years, FTY720 has emerged as a key player in cancer therapy. FTY720 has shown preclinical antitumor efficacy in cancer models, including those of breast, bladder, glioblastoma, ovarian, prostate, lung, liver and hematopoietic malignancies. In these malignancies, the anticancer effects of FTY720 are reportedly attributed to its cytotoxicity towards cancer cells through caspase-dependent, caspase-independent or autophagic cell death pathways. Moreover, in most instances, phosphorylation of FTY720 is not required for its cytotoxic effect. To date, numerous molecular targets have been proposed for the unphosphorylated form of FTY720, including ROS, PP2A, cyclin D1, SphK1 and 14-3-3. These downstream targets appear to be associated with the ability of FTY720 to suppress cell growth and/or induce cell death. These observations make FTY720 an attractive therapeutic drug for developing alternative treatment protocols, and, possibly, for combining with other anticancer drugs to overcome drug resistance and achieve better outcomes. Thus, it is clear that further studies are required to elucidate the full spectrum of direct and downstream cellular targets of this FTY720. Ultimately, novel molecular targets will be found and may hold promise for clinical cancer therapy.

\section{Acknowledgements}

The present study was supported by grants from the Key Subject of Jiangsu Province (X4200722).

\section{References}

1. Billich A, Bornancin F, Dévay P, Mechtcheriakova D, Urtz N and Baumruker T: Phosphorylation of the immunomodulatory drug FTY720 by sphingosine kinases. J Biol Chem 278: 47408-47415, 2003.

2. Paugh SW, Payne SG, Barbour SE, Milstien S and Spiegel S: The immunosuppressant FTY720 is phosphorylated by sphingosine kinase type 2. FEBS Lett 554: 189-193, 2003.

3. Suzuki S, Enosawa S, Kakefuda T, et al: A novel immunosuppressant, FTY720, with a unique mechanism of action, induces long-term graft acceptance in rat and dog allotransplantation. Transplantation 61: 200-205, 1996.

4. Enosawa S, Suzuki S, Kakefuda T, Li XK and Amemiya H: Induction of selective cell death targeting on mature T-lymphocytes in rats by a novel immunosuppressant, FTY720. Immunopharmacology 34: 171-179, 1996.

5. Suzuki S, Li XK, Shinomiya T, et al: Induction of lymphocyte apoptosis and prolongation of allograft survival by FTY720. Transplant Proc 28: 2049-2050, 1996.

6. Suzuki S, Enosawa S, Kakefuda T, Amemiya H, Hoshino Y and Chiba K: Long-term graft acceptance in allografted rats and dogs by treatment with a novel immunosuppressant, FTY720. Transplant Proc 28: 1375-1376, 1996.

7. Suzuki S, Enosawa S, Kakefuda T, et al: Immunosuppressive effect of a new drug, FTY720, on lymphocyte responses in vitro and cardiac allograft survival in rats. Transplant Immunol 4: 252-255, 1996.

8. Pitman MR, Woodcock JM, Lopez AF and Pitson SM: Molecular targets of FTY720 (fingolimod). Curr Mol Med 12: 1207-1219, 2012.

9. Azuma H, Takahara S, Ichimaru N, et al: Marked prevention of tumor growth and metastasis by a novel immunosuppressive agent, FTY720, in mouse breast cancer models. Cancer Res 62: 1410-1419, 2002.

10. Sonoda Y, Yamamoto D, Sakurai S, et al: FTY720, a novel immunosuppressive agent, induces apoptosis in human glioma cells. Biochem Biophys Res Commun 281: 282-288, 2001.

11. Chua CW, Lee DT, Ling MT, et al: FTY720, a fungus metabolite, inhibits in vivo growth of androgen-independent prostate cancer. Int J Cancer 117: 1039-1048, 2005.

12. Schmid G, Guba M, Papyan A, et al: FTY720 inhibits tumor growth and angiogenesis. Transplant Proc 37: 110-111, 2005.

13. Zhang N, Qi Y, Wadham C, et al: FTY720 induces necrotic cell death and autophagy in ovarian cancer cells: a protective role of autophagy. Autophagy 6: 1157-1167, 2010.

14. Lee TK, Man K, Ho JW, et al: FTY720 induces apoptosis of human hepatoma cell lines through PI3-K-mediated Akt dephosphorylation. Carcinogenesis 25: 2397-2405, 2004.

15. Vadas M, Xia P, McCaughan G and Gamble J: The role of sphingosine kinase 1 in cancer: oncogene or non-oncogene addiction? Biochim Biophys Acta 1781: 442-447, 2008.

16. Wang JD, Takahara S, Nonomura N, et al: Early induction of apoptosis in androgen-independent prostate cancer cell line by FTY720 requires caspase-3 activation. Prostate 40: 50-55, 1999.

17. Hu S, Vincenz C, Buller M and Dixit VM: A novel family of viral death effector domain-containing molecules that inhibit both CD-95- and tumor necrosis factor receptor-1-induced apoptosis. J Biol Chem 272: 9621-9624, 1997.

18. Cohen GM: Caspases: the executioners of apoptosis. Biochem J 326 (Pt 1): 1-16, 1997.

19. Zheng T, Meng X, Wang J, et al: PTEN- and p53-mediated apoptosis and cell cycle arrest by FTY720 in gastric cancer cells and nude mice. J Cell Biochem 111: 218-228, 2010.

20. Ubai T, Azuma H, Kotake Y, et al: FTY720 induced Bcl-associated and Fas-independent apoptosis in human renal cancer cells in vitro and significantly reduced in vivo tumor growth in mouse xenograft. Anticancer Res 27: 75-88, 2007.

21. Yasui H, Hideshima T, Raje N, et al: FTY720 induces apoptosis in multiple myeloma cells and overcomes drug resistance. Cancer Res 65: 7478-7484, 2005. 
22. Susin SA, Lorenzo HK, Zamzami N, et al: Molecular characterization of mitochondrial apoptosis-inducing factor. Nature 397: 441-446, 1999.

23. Liu Q, Alinari L, Chen CS, et al: FTY720 shows promising in vitro and in vivo preclinical activity by downmodulating Cyclin D1 and phospho-Akt in mantle cell lymphoma. Clin Cancer Res 16: 3182-3192, 2010.

24. Liu Q, Zhao X, Frissora F, et al: FTY720 demonstrates promising preclinical activity for chronic lymphocytic leukemia and lymphoblastic leukemia/lymphoma. Blood 111: 275-284, 2008.

25. Wallington-Beddoe CT, Hewson J, Bradstock KF and Bendall LJ: FTY720 produces caspase-independent cell death of acute lymphoblastic leukemia cells. Autophagy 7: 707-715, 2011.

26. Liao A, Hu R, Zhao Q, et al: Autophagy induced by FTY720 promotes apoptosis in U266 cells. Eur J Pharm Sci 45: 600-605, 2012.

27. Alinari L, Baiocchi RA and Praetorius-Ibba M: FTY720 induced blockage of autophagy enhances anticancer efficacy of milatuzumab in mantle cell lymphoma: is FTY720 the next autophagy-blocking agent in lymphoma treatment? Autophagy 8 : 416-417, 2012.

28. Alinari L, Mahoney E, Patton J, et al: FTY720 increases CD74 expression and sensitizes mantle cell lymphoma cells to milatuzumab-mediated cell death. Blood 118: 6893-6903, 2011.

29. Levine B and Kroemer G: Autophagy in the pathogenesis of disease. Cell 132: 27-42, 2008

30. Levine B and Yuan J: Autophagy in cell death: an innocent convict? J Clin Invest 115: 2679-2688, 2005.

31. Ogata M, Hino S, Saito A, et al: Autophagy is activated for cell survival after endoplasmic reticulum stress. Mol Cell Biol 26 : 9220-9231, 2006.

32. Wirawan E, Vanden Berghe T, Lippens S, Agostinis P and Vandenabeele P: Autophagy: for better or for worse. Cell Res 22: 43-61, 2012.

33. Estrada-Bernal A, Palanichamy K, Ray Chaudhury A and Van Brocklyn JR: Induction of brain tumor stem cell apoptosis by FTY720: a potential therapeutic agent for glioblastoma. Neuro Oncol 14: 405-415, 2012

34. Ng KT, Man K, Ho JW, et al: Marked suppression of tumor growth by FTY720 in a rat liver tumor model: The significance of downregulation of cell survival Akt pathway. Int J Oncol 30 375-380, 2007

35. Zhou C, Ling MT, Kin-Wah Lee T, Man K, Wang X and Wong YC: FTY720, a fungus metabolite, inhibits invasion ability of androgen-independent prostate cancer cells through inactivation of RhoA-GTPase. Cancer Lett 233: 36-47, 2006.

36. Kappos L, Radue EW, O'Connor P, et al: A placebo-controlled trial of oral fingolimod in relapsing multiple sclerosis. N Engl J Med 362: 387-401, 2010.

37. Azuma H, Takahara S, Horie S, Muto S, Otsuki Y and Katsuoka Y: Induction of apoptosis in human bladder cancer cells in vitro and in vivo caused by FTY720 treatment. J Urol 169: 2372-2377, 2003.

38. Devasagayam TP, Tilak JC, Boloor KK, Sane KS, Ghaskadbi SS and Lele RD: Free radicals and antioxidants in human health: current status and future prospects. J Assoc Physicians India 52: 794-804, 2004

39. Hung JH, Lu YS, Wang YC, et al: FTY720 induces apoptosis in hepatocellular carcinoma cells through activation of protein kinase C delta signaling. Cancer Res 68: 1204-1212, 2008.

40. Omar HA, Chou CC, Berman-Booty LD, et al: Antitumor effects of OSU-2S, a nonimmunosuppressive analogue of FTY720, in hepatocellular carcinoma. Hepatology 53: 1943-1958, 2011.

41. Perrotti D and Neviani P: Protein phosphatase 2A: a target for anticancer therapy. Lancet Oncol 14: e229-e238, 2013.

42. Yin X, Zhang N and Di W: Regulation of LC3-dependent protective autophagy in ovarian cancer cells by protein phosphatase 2A. Int J Gynecol Cancer 23: 630-641, 2013.

43. Yang Y, Huang Q, Lu Y, Li X and Huang S: Reactivating PP2A by FTY720 as a novel therapy for AML with C-KIT tyrosine kinase domain mutation. J Cell Biochem 113: 1314-1322, 2012.

44. Saddoughi SA, Gencer S, Peterson YK, et al: Sphingosine analogue drug FTY720 targets I2PP2A/SET and mediates lung tumour suppression via activation of PP2A-RIPK1-dependent necroptosis. EMBO Mol Med 5: 105-121, 2013.

45. Neviani P, Santhanam R, Trotta R, et al: The tumor suppressor PP2A is functionally inactivated in blast crisis CML through the inhibitory activity of the BCR/ABL-regulated SET protein. Cancer Cell 8: 355-368, 2005.
46. Neviani P, Santhanam R, Oaks JJ, et al: FTY720, a new alternative for treating blast crisis chronic myelogenous leukemia and Philadelphia chromosome-positive acute lymphocytic leukemia. J Clin Invest 117: 2408-2421, 2007.

47. Cristobal I, Garcia-Orti L, Cirauqui C, Alonso MM, Calasanz MJ and Odero MD: PP2A impaired activity is a common event in acute myeloid leukemia and its activation by forskolin has a potent anti-leukemic effect. Leukemia 25: 606-614, 2011.

48. Roberts KG,Smith AM,McDougall F, et al: Essential requirement for PP2A inhibition by the oncogenic receptor c-KIT suggests $\mathrm{PP} 2 \mathrm{~A}$ reactivation as a strategy to treat $\mathrm{c}-\mathrm{KIT}^{+}$cancers. Cancer Res 70: 5438-5447, 2010.

49. Manning G, Whyte DB, Martinez R, Hunter T and Sudarsanam S: The protein kinase complement of the human genome. Science 298: 1912-1934, 2002.

50. Nagaoka Y, Otsuki K, Fujita T and Uesato S: Effects of phosphorylation of immunomodulatory agent FTY720 (fingolimod) on antiproliferative activity against breast and colon cancer cells. Biol Pharm Bull 31: 1177-1181, 2008.

51. Lee YJ, Kim NY, Suh YA and Lee C: Involvement of ROS in curcumin-induced autophagic cell death. Korean J Physiol Pharmacol 15: 1-7, 2011.

52. Liu JM, Pan F, Li L, et al: Piperlongumine selectively kills glioblastoma multiforme cells via reactive oxygen species accumulation dependent JNK and p38 activation. Biochem Biophys Res Commun 437: 87-93, 2013.

53. Borders EB, Bivona C and Medina PJ: Mammalian target of rapamycin: biological function and target for novel anticancer agents. Am J Health Syst Pharm 67: 2095-2106, 2010.

54. Yap TA, Garrett MD, Walton MI, Raynaud F, de Bono JS and Workman P: Targeting the PI3K-AKT-mTOR pathway: progress, pitfalls, and promises. Curr Opin Pharmacol 8: 393-412, 2008

55. LoPiccolo J, Blumenthal GM, Bernstein WB and Dennis PA: Targeting the PI3K/Akt/mTOR pathway: effective combinations and clinical considerations. Drug Resist Updat 11: 32-50, 2008

56. Alvarez M, Roman E, Santos ES and Raez LE: New targets for non-small-cell lung cancer therapy. Expert Rev Anticancer Ther 7: 1423-1437, 2007.

57. Rosell R, Felip E, Garcia-Campelo R and Balana C: The biology of non-small-cell lung cancer: identifying new targets for rational therapy. Lung Cancer 46: 135-148, 2004.

58. Zheng J, Zou X and Yao J: The antitumor effect of GDC-0941 alone and in combination with rapamycin in breast cancer cells. Chemotherapy 58: 273-281, 2012.

59. Liao WT, Li TT, Wang ZG, et al: MicroRNA-224 promotes cell proliferation and tumor growth in human colorectal cancer by repressing PHLPP1 and PHLPP2. Clin Cancer Res 19: 4662-4672, 2013

60. Yothaisong S, Dokduang H, Techasen A, et al: Increased activation of PI3K/AKT signaling pathway is associated with cholangiocarcinoma metastasis and $\mathrm{PI} 3 \mathrm{~K} / \mathrm{mTOR}$ inhibition presents a possible therapeutic strategy. Tumour Biol: Jul 6, 2013 (Epub ahead of print)

61. Wu P and $\mathrm{Hu}$ YZ: PI3K/Akt/mTOR pathway inhibitors in cancer: a perspective on clinical progress. Curr Med Chem 17: 4326-4341, 2010

62. Ghayad SE and Cohen PA: Inhibitors of the PI3K/Akt/mTOR pathway: new hope for breast cancer patients. Recent Pat Anticancer Drug Discov 5: 29-57, 2010.

63. Cortot A, Armand JP and Soria JC: PI3K-AKT-mTOR pathway inhibitors. Bull Cancer 93: 19-26, 2006 (In French).

64. Chua CW, Chiu YT, Yuen HF, et al: Suppression of androgenindependent prostate cancer cell aggressiveness by FTY720: validating Runx2 as a potential antimetastatic drug screening platform. Clin Cancer Res 15: 4322-4335, 2009.

65. Li MH, Hla T and Ferrer F: FTY720 inhibits tumor growth and enhances the tumor-suppressive effect of topotecan in neuroblastoma by interfering with the sphingolipid signaling pathway. Pediatr Blood Cancer 60: 1418-1423, 2013.

66. Chang CY, Ma KH, Wang JK, Tung YL and Chueh SH: Inhibition of protein kinase $\mathrm{C}$ promotes differentiation of neuroblastoma x glioma NG108-15 hybrid cells. Eur J Neurosci 34: 1074-1084, 2011.

67. Weng JR, Bai LY, Chiu CF, Hu JL, Chiu SJ and Wu CY: Cucurbitane triterpenoid from Momordica charantia induces apoptosis and autophagy in breast cancer cells, in part, through peroxisome proliferator-activated receptor gamma activation. Evid Based Complement Alternat Med 2013: 935675, 2013. 
68. Tolba MF, Esmat A, Al-Abd AM, et al: Caffeic acid phenethyl ester synergistically enhances docetaxel and paclitaxel cytotoxicity in prostate cancer cells. IUBMB Life 65: 716-729, 2013.

69. Li X, Pu J, Jiang S, et al: Henryin, an ent-kaurane diterpenoid, inhibits Wnt signaling through interference with beta-catenin/TCF4 interaction in colorectal cancer cells. PLoS One 8: e68525, 2013.

70. Chung YC, Lu LC, Tsai MH, et al: The inhibitory effect of ellagic acid on cell growth of ovarian carcinoma cells. Evid Based Complement Alternat Med 2013: 306705, 2013.

71. Li H, Sun L, Xu Y, et al: Overexpression of MTA3 correlates with tumor progression in non-small cell lung cancer. PLoS One 8: e66679, 2013.

72. Chang MS, Kim DH, Roh JK, et al: Epstein-Barr virus-encoded BARF1 promotes proliferation of gastric carcinoma cells through regulation of NF-kappaB. J Virol 87: 10515-10523, 2013.

73. Jiang J, Huang X, Wang Y, Deng A and Zhou J: FTY720 induces cell cycle arrest and apoptosis of rat glomerular mesangial cells. Mol Biol Rep 39: 8243-8250, 2012.

74. Li QY, Chi YY and Liu SQ: Cell cycle arrest effects of large-dose FTY720 on lymphocytes in mouse skin transplantation models. Immunopharmacol Immunotoxicol 30: 365-381, 2008.

75. Gulappa T, Reddy RS, Suman S, Nyakeriga AM and Damodaran C: Molecular interplay between cdk4 and p21 dictates G/G cell cycle arrest in prostate cancer cells. Cancer Lett 337: 177-183, 2013

76. Bermudez O, Hennen E, Koch I, Lindner M and Eickelberg O Gli1 mediates lung cancer cell proliferation and Sonic Hedgehogdependent mesenchymal cell activation. PLoS One 8: e63226, 2013.

77. Aigelsreiter A, Ress AL, Bettermann K, et al: Low expression of the putative tumour suppressor spinophilin is associated with higher proliferative activity and poor prognosis in patients with hepatocellular carcinoma. Br J Cancer 108: 1830-1837, 2013.

78. Guo J, Gao J, Li Z, et al: Adenovirus vector-mediated Gli1 siRNA induces growth inhibition and apoptosis in human pancreatic cancer with Smo-dependent or Smo-independent Hh pathway activation in vitro and in vivo. Cancer Lett 339: 185-194, 2013.

79. Yoon JS, Kim HM, Yadunandam AK, et al: Neferine isolated from Nelumbo nucifera enhances anti-cancer activities in Hep3B cells: Molecular mechanisms of cell cycle arrest, ER stress induced apoptosis and anti-angiogenic response. Phytomedicine 20: 1013-1022, 2013

80. Kurokawa K, Akaike Y, Masuda K, et al: Downregulation of serine/arginine-rich splicing factor 3 induces G1 cell cycle arres and apoptosis in colon cancer cells. Oncogene: March 18, 2013 (Epub ahead of print).

81. Xing Z, Zhang Y, Zhang X, Yang Y, Ma Y and Pang D Fangchinoline induces G1 arrest in breast cancer cells through cell-cycle regulation. Phytother Res: Feb 11, 2013 (Epub ahead of print).

82. Steck PA, Pershouse MA, Jasser SA, et al: Identification of a candidate tumour suppressor gene, MMAC1, at chromosome 10q23.3 that is mutated in multiple advanced cancers. Nat Genet 15: 356-362, 1997.
83. Santoni M, Burattini L, Nabissi M, et al: Essential role of gli proteins in glioblastoma multiforme. Curr Protein Pept Sci 14: 133-140, 2013.

84. Yang P, Wang Y, Peng X, et al: Management and survival rates in patients with glioma in China (2004-2010): a retrospective study from a single-institution. J Neurooncol 113: 259-266, 2013.

85. Brucka A and Szyłł K: Immunoexpression of the PTEN protein and matrix metalloproteinase-2 in endometrial cysts, endometrioid and clear cell ovarian cancer. Ginekol Pol 84: 344-351, 2013.

86. Abdulkareem IH and Blair M: Effects of indomethacin on expression of PTEN tumour suppressor in human cancers. Niger Med J 54: 100-106, 2013.

87. McQuitty E, Zhang W, Hendrickson H, et al: Lung adenocarcinoma biomarker incidence in Hispanic versus non-Hispanic white patients. Arch Pathol Lab Med: Jun 26, 2013 (Epub ahead of print).

88. Filippini SE and Vega A: Breast cancer genes: beyond BRCA1 and BRCA2. Front Biosci 18: 1358-1372, 2013.

89. Alshaker H, Sauer L, Monteil D, et al: Therapeutic potential of targeting SK1 in human cancers. Adv Cancer Res 117: 143-200, 2013.

90. Lim KG, Tonelli F, Berdyshev E, et al: Inhibition kinetics and regulation of sphingosine kinase 1 expression in prostate cancer cells: functional differences between sphingosine kinase 1a and 1b. Int J Biochem Cell Biol 44: 1457-1464, 2012.

91. Rosa R, Marciano R, Malapelle U, et al: Sphingosine kinase 1 overexpression contributes to cetuximab resistance in human colorectal cancer models. Clin Cancer Res 19: 138-147, 2013.

92. Lim KG, Tonelli F, Li Z, et al: FTY720 analogues as sphingosine kinase 1 inhibitors: enzyme inhibition kinetics, allosterism, proteasomal degradation, and actin rearrangement in MCF-7 breast cancer cells. J Biol Chem 286: 18633-18640, 2011.

93. Tonelli F, Lim KG, Loveridge C, et al: FTY720 and (S)-FTY720 vinylphosphonate inhibit sphingosine kinase 1 and promote its proteasomal degradation in human pulmonary artery smooth muscle, breast cancer and androgen-independent prostate cancer cells. Cell Signal 22: 1536-1542,2010.

94. Pitson SM: Regulation of sphingosine kinase and sphingolipid signaling. Trends Biochem Sci 36: 97-107, 2011.

95. Li MH, Hla T and Ferrer F: Sphingolipid modulation of angiogenic factor expression in neuroblastoma. Cancer Prev Res (Phila) 4: 1325-1332, 2011

96. Xu Z, Graham K, Foote M, et al: 14-3-3 targets chaperoneassociated misfolded proteins to aggresomes. J Cell Sci 126 4173-4186.

97. Zhang L, Chen J and Fu H: Suppression of apoptosis signalregulating kinase 1-induced cell death by 14-3-3 proteins. Proc Natl Acad Sci USA 96: 8511-8515, 1999.

98. Woodcock JM, Ma Y, Coolen C, et al: Sphingosine and FTY720 directly bind pro-survival 14-3-3 proteins to regulate their function. Cell Signal 22: 1291-1299, 2010. 